\title{
Influencia de los sucesos perinatales en la incidencia de la mortalidad infantil en Costa Rica en el periodo 2006-2010
}

\author{
Ever Zeledón Díaz
}

Institución: Universidad de Costa Rica

Maestría en Enfermería Ginecológica, Obstétrica y Perinatal

\section{CÓMO CITAR}

Zeledón, E. (2012) Influencia de los sucesos perinatales en la incidencia de la mortalidad infantil en Costa Rica en el periodo 2006-2010. Rev. Enfermería Actual en Costa Rica, 23, 1-11 Recuperado de: http://www. revenf.ucr.ac.cr/mortalidadinfantil.pdf ISSN 1409-4568

\section{RESUMEN}

El objetivo de este artículo es presentar los resultados de una revisión documental sobre la influencia de sucesos perinatales, esto es, hechos ocurridos en el periodo perinatal, en el comportamiento de la mortalidad infantil en Costa Rica durante el quinquenio 2006-2010. Se trata de un estudio descriptivo y retrospectivo. La población estuvo conformada por los menores de un año fallecidos durante el período referido. Se realizó una comparación, a partir de la tasa de mortalidad infantil, por sexo y provincia. Como resultado, se evidenció que la tasa de mortalidad infantil en nuestro país posee una tendencia a la disminución y que los sucesos perinatales son la causa de la mayor cantidad de muertes. Se concluye que, en Costa Rica, durante el periodo 2006-2010, la mortalidad infantil se produjo por causas perinatales en su mayoría. Como principales factores de riesgo se encuentran los trastornos respiratorios, los cardiovasculares y aquellos relacionados con la duración de la gestación y el crecimiento fetal, por ejemplo, el Síndrome de dificultad respiratoria y la prematuridad.

Palabras clave: Factores de riesgo, mortalidad infantil, sucesos perinatales.

\footnotetext{
${ }^{1}$ Fecha de recepción: 20 de junio del 2012

Fecha de aceptación: 3 de Setiembre del 2012

${ }^{2}$ Licenciado en Enfermería. Enfermero en el Hospital Rafael Angel Calderón Guardia. Estudiante de la Maestría en Enfermería Ginecológica, Obstétrica y Perinatal. Correo electrónico: adez282@,hotmail.com
} 
. $\left(\frac{10}{20}\right.$

(1)

Revista Electrónica Enfermería Actual en Costa Rica

www.revenf.ucr.ac.cr

\title{
Influence of events perinatal incidence of infant mortality in Costa Rica in the period 2006- $2010^{1}$
}

Ever Zeledón Díaz ${ }^{2}$

Institution: University of Costa Rica

Master in Nursing Gynecologic, Obstetric and Perinatal

\section{CITED}

Zeledón, E. (2012) Influence of events perinatal incidence of infant mortality in Costa Rica in the period 20062010. Rev. Enfermería Actual en Costa Rica, 23, 1-12. Available: http://www. revenf.ucr.ac.cr/ mortalidadinfantil.pdf ISSN 1409-4568

\begin{abstract}
The objective of this paper is to present the results of a literature review on the influence of perinatal events, defined as events in the perinatal period, the behavior of infant mortality in Costa Rica, during the 2006-2010 periods. It is a descriptive study. The population consisted of those who died under one year occurred in the period in question. A comparison was made, from the infant mortality rate by sex and province. Results evidenced that the infant mortality rate in our country, has a tendency to decrease. Moreover, the events provided perinatal death as much. We conclude that in Costa Rica in the period 2006-2010, infant mortality is expressed mostly perinatal causes, and as major risk factors be appointing the respiratory, cardiovascular, related to length of gestation and growth fetal, including in these respiratory distress syndrome and prematurity.
\end{abstract}

Key words: Risk factors, infant mortality, perinatal events.

\footnotetext{
${ }^{1}$ Date of reception: Junio 20, 2012

Date of acceptance: September 3, 2012

${ }^{2}$ Degree in Nursing. He Works at the Rafael Ángel Calderón Guardia Hospital. Student of the Master of Nursing Gynecologic,

Obstetric and Perinatal. University of Costa Rica. E mail: adez282@hotmail.com
} 


\section{INTRODUCCIÓN}

La muerte perinatal es un tema prioritario para la salud. La Organización Mundial de la Salud (2005) afirma que "la vida requiere un buen comienzo mucho antes de nacer, pero es justo antes del parto, durante él y en las primeras horas y días de existencia cuando corre mayor peligro" (p.85); esto denota la vulnerabilidad de este periodo.

Además, Mejía (2000), en referencia a este lapso, y la oportunidad de supervivencia, menciona que, “...está influenciado por un gran número de factores ambientales, sociales y genéticos los cuales pueden determinar el crecimiento fetal, riesgo de malformaciones, parto prematuro, bajo peso al nacer o la utilización de servicios de neonatología y obstetricia”. (p.105) y así mismo, la mortalidad infantil.

La mortalidad infantil se define como "la desaparición permanente de todo tipo de signo de vida, antes de cumplir el primer año de vida” (Mejía, 2000, p.105). Esta se expresa mediante la tasa de mortalidad infantil, la cual consiste en la relación entre las defunciones de menores de un año ocurridas durante un año calendario y los nacimientos ocurridos en el mismo período, por cada mil nacimientos.

Mejía (2000) también señala que la mortalidad neonatal es un indicador utilizado para expresar el riesgo de fallecer o la expectativa de vida de un recién nacido, en los primeros 28 días de vida, en un determinado país. En Costa Rica, para el año 2010, las defunciones neonatales representaron el 72,1\%, lo cual resultó en una tasa de defunción neonatal de 6,82 por cada mil. Esta muestra un aumento de un 0,38 con respecto al año 2009, en el que fue de 6,44 por cada mil. (INEC, 2011)

Así mismo, como parte de la mortalidad neonatal se encuentra la perinatal, es decir, la muerte ocurrida durante el período que se extiende desde las 22 semanas de gestación hasta los 7 días completos posteriores al nacimiento.

Entre las principales causas de muerte perinatal, Salgado (2010) señala las siguientes: la asfixia intrauterina, el bajo peso al nacer, el traumatismo de parto y las infecciones intrauterinas y neonatales.

Ticona (2005), por su parte, refiere que:

...cada año en el mundo nacen muertos alrededor de 4,3 millones de niños y 3,3 millones mueren en la primera semana de vida, de estos 7,6 millones de muertes perinatales, el 98\% ocurren en países en vías de desarrollo. La mortalidad perinatal se ha mantenido sin cambios en las últimas 


\section{Revista Electrónica Enfermería Actual en costa Rica}

décadas y ocupa el primer lugar como causa de muerte infantil, por lo que debe ser considerada como problema prioritario de Salud Pública. (p. 314)

Aunado a lo anterior, la trascendencia de la tasa de mortalidad infantil como indicador de salud y bienestar social es bien conocida, además de ser indicador de desarrollo de un país.

Según datos obtenidos por el Instituto Nacional de Estadística y Censo (2011):

...la tasa de mortalidad infantil para el 2010 fue de 9,46 por mil. Esto significa que por cada mil nacimientos fallecieron aproximadamente 9 menores de un año. Al compararla con la tasa del año 2009 (8,84 por mil), presenta un aumento de 0,62 por mil. (p. 1$)$

Del total de muertes de menores de un año, las defunciones neonatales, es decir, las ocurridas en los primeros 28 días de vida, representan el 72,1\%. Esto resulta en una tasa de defunción neonatal de 6,82 por cada mil, la cual muestra un aumento de un 0,38 con respecto al año 2009 , en el que fue de 6,44 por cada mil.

Vargas (2001) afirma que, al comparar la mortalidad infantil entre Costa Rica y otros países, como Canadá y Cuba, nuestra nación muestra un aumento de muertes; la brecha más importante se encuentra en las afecciones perinatales. Lo anterior manifiesta la importancia de los sucesos perinatales en la incidencia de la mortalidad infantil. Esta última, si bien ha disminuido en las últimas décadas debido a las mejoras en el tratamiento de las infecciones y enfermedades parasitarias, tal y como lo afirma Vargas (2001), los factores perinatales continúan significando un reto en su prevención y manejo, lo cual causa un aumento en la mortalidad infantil.

Cabe resaltar que en Costa Rica no se han realizado estudios previos sobre la incidencia de los sucesos perinatales en la mortalidad infantil, esto incluye la mortalidad perinatal.

El objetivo de esta revisión es determinar la influencia de los sucesos perinatales en la incidencia de la mortalidad infantil en Costa Rica durante el periodo 2006-2010. Este estudio es motivado por el interés que tiene el autor en presentar una realidad que debe ser valorada por la relación que tiene con la intervención que brinda el personal de enfermería ginecoobstétrica y perinatal.

\section{MATERIALES Y MÉTODOS}


El universo de estudio estuvo conformado por los fallecidos menores de un año durante el período referido. Se realizó una comparación, a partir de la tasa de mortalidad infantil, por sexo y provincia. Se efectuó una recopilación acerca de los índices y tasas de mortalidad infantil en Costa Rica, mediante la base de datos del Instituto Nacional de Estadística y Censo de Costa Rica, y sobre la mortalidad infantil ocurrida durante el periodo que abarca desde el 1 de enero de 2006 hasta el 31 de diciembre del 2010.

Para la recolección de información, se desarrolló una matriz de datos que contenía las siguientes variables de interés: sexo, causas de mortalidad infantil y factores de riesgo perinatales. Además, se efectuó un análisis, por provincias, con el fin de observar la tendencia por sectores. Posteriormente, se tabularon los datos y presentaron en gráficos diseñados en el programa Microsoft Excel.

\section{Consideraciones Éticas}

En este estudio de revisión, se contemplaron y se respetaron los derechos de autor; no se realizaron alteraciones a los datos extraídos de cada publicación. Además, se evidenció el aporte de cada autor en las citas mencionadas.

\section{RESULTADOS}

En el período del 2006 al 2010, hubo un total de 365544 nacimientos con 3434 defunciones; la tasa de mortalidad infantil (TMI) osciló entre 10,05 y 8,84 por 1000 nacidos vivos.

Geográficamente, la provincia de Puntarenas registró la tasa de mortalidad infantil más alta en este quinquenio -con una TMI promedio de 10,66 por cada mil. Las provincias de Limón, San José, Guanacaste y Cartago le siguen, con un promedio TMI de 10,32, 9,80, 9,69 y 9,56, respectivamente. Finalmente, Alajuela mostró una de las TMI más baja -8.32)-, solo superada por Heredia con 7,32.

Con respecto a la variable "sexo", mostró el mismo comportamiento que en años anteriores; en Costa Rica, la TMI, es más alta en los niños que en las niñas. Este patrón se repite por primera vez, desde el año 2006, en todas las provincias del país. Véase en el gráfico 1 la tasa de mortalidad infantil en los últimos 5 años. 


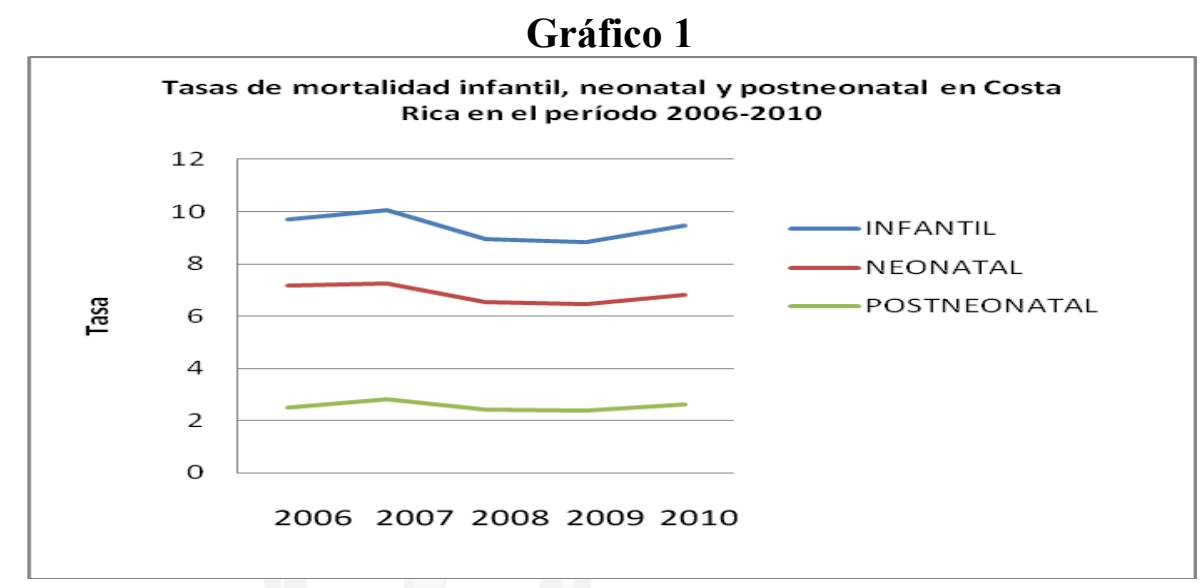

Como se observa en el gráfico 1, la mortalidad infantil en los últimos cinco años ha disminuido, sin embargo, durante el año 2011 se produjo un aumento del 0,62 por cada mil nacidos con respecto al año 2009 .

Además, es posible evidenciar cómo la mortalidad neonatal incide, en mayor medida, sobre la mortalidad infantil. Esto último otorga un punto de referencia a partir del cual se concluye que se debe evaluar los servicios de salud y enfocar la atención en estos.

En el gráfico 2, se muestran las principales causas de muerte infantil en Costa Rica en los últimos cinco años.

\section{Gráfico 2}

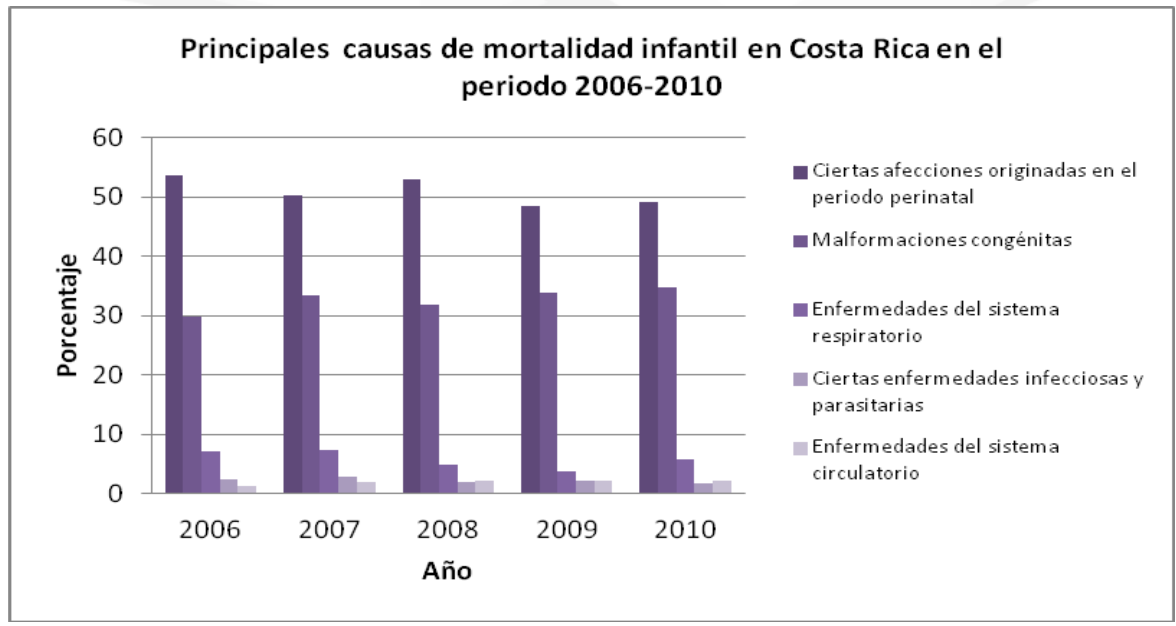

Fuente: Gráfico realizado a partir de datos suministrados por el INEC 
En el gráfico anterior, se evidencia cómo las causas de la mortalidad infantil han mantenido una constante; el componente perinatal ocupó más del $50 \%$ de la mortalidad infantil, con 1748 defunciones en todo el período, mientras que las malformaciones congénitas ocuparon el segundo lugar como agente causal de la mortalidad infantil. Finalmente, se encuentran las enfermedades respiratorias y las de índole infecciosa y parasitaria.

Este patrón ha persistido en los últimos años, aunque se visualiza una tendencia de incremento en la cantidad de casos por malformaciones congénitas; así lo afirma Ávila (2007). Nótese este aspecto en el gráfico 3.

\section{Gráfico 3}

\section{Distribución porcentual de defunciones infantiles según causa de muerte $2005-2010$}

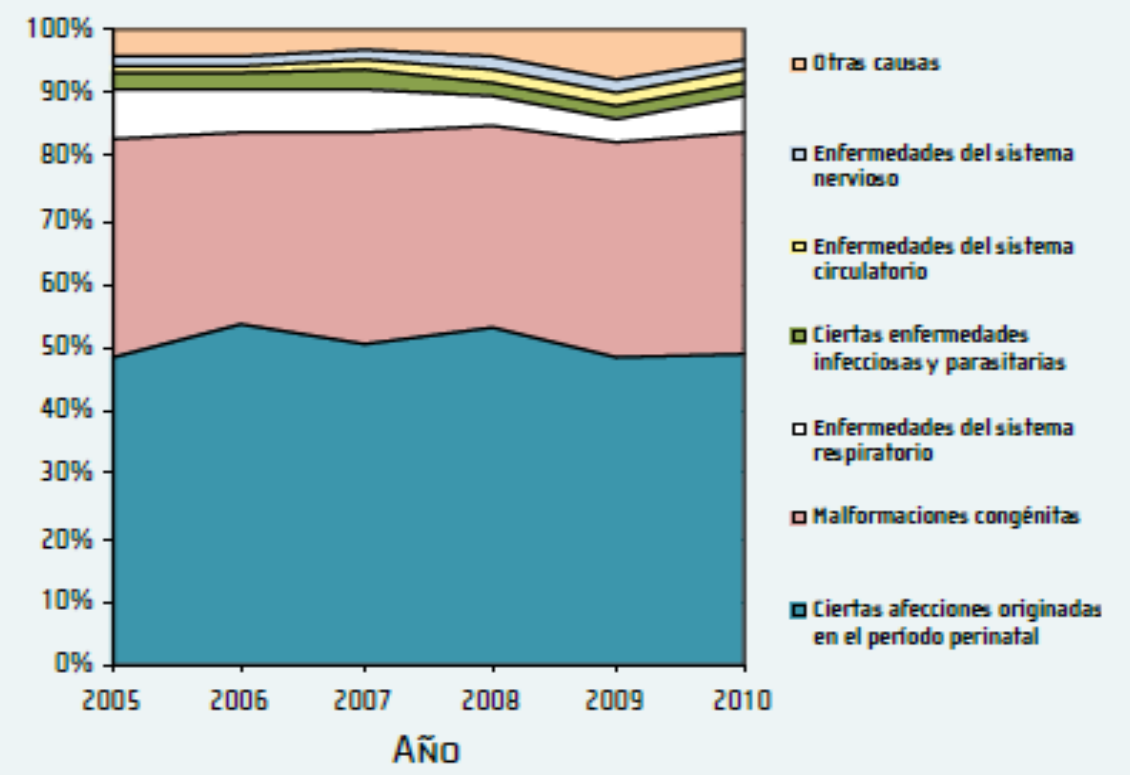

Fuente: INEC, 2010

Como se observa en este gráfico, las causas perinatales son el principal factor de mortalidad infantil; según el INEC, en el año 2006 las principales causas de muerte se originaron durante el periodo perinatal, $53,76 \%$. Dentro de este grupo, el mayor incremento se visualiza en los trastornos respiratorios y cardiovasculares, con 45,97\%, seguido por los trastornos relacionados con la duración de la gestación y el crecimiento fetal con $16,40 \%$.

En el 2007, 2008 y 2009, esta situación se mantiene igual. Si bien para el año 2010 las principales causas de muerte infantil siguen siendo originadas en el periodo perinatal, el INEC afirma que las defunciones por 
.

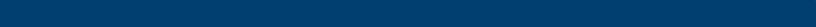

prematuridad extrema, con $20,61 \%$, seguido por el Síndrome de dificultad respiratoria del recién nacido, con $12,42 \%$, son las principales afecciones que inciden en la mortalidad infantil.

\section{DISCUSIÓN}

En Costa Rica y en el resto del mundo, la disminución de las cifras de la mortalidad infantil ha sido evidente en los últimos años. Como lo muestra el Ministerio de Planificación Nacional y Política Económica (2011), para el año 1990 los casos de mortalidad infantil fueron de 1211, con una tasa de mortalidad infantil de 14,8, mientras que, dos 20 años después, la TMI disminuyó a 9,4. Kaempffer y Medina (2000) refieren que los valores de mortalidad infantil de un país están ligados tanto a factores de tipo económico y social como a los servicios de atención de salud existentes. Si bien Costa Rica ha mostrado un gran desarrollo económico en las últimas décadas, también ha sufrido un importante avance tecnológico que permite brindar una mejor atención a la mujer embarazada y al recién nacido. Lo anterior evidencia la existencia de un sistema de salud que se encuentra anuente a acoplarse al cambio. Los autores antes mencionados refieren que:

...hoy en día se dan condiciones favorables para alcanzar nuevos logros. Aunque no hay evidencias claras

de que la gran mayoría de los niños sean deseados, se puede apreciar que las actividades de promoción y

protección de la salud se realizan adecuadamente. (s.p.)

Lo anterior alude a cómo, desde el primer nivel de atención en servicios médicos se puede prevenir la mortalidad infantil mediante la promoción de la salud y prevención de la enfermedad. De igual manera,, mejorar la consulta prenatal y realizar una categorización de riesgo según la historia clínica, permitirá brindar una atención que prevenga efectos adversos que puedan desencadenar complicaciones y causar la muerte del recién nacido.

Con respecto a la epidemiología de la mortalidad infantil, se logra evidenciar un cambio; hace unas décadas, las enfermedades infecciosas eran las principales causas de muerte infantil. En la actualidad, las afecciones originadas en el período perinatal producen la mayor cantidad de muertes en niños menores de un año; entre estas se incluyen los s trastornos relacionados con la duración de la gestación y el crecimiento fetal.

En Costa Rica, al igual que en países como Chile, la situación de la mortalidad neonatal temprana se relaciona estrechamente con las causas de muerte, pues las principales afecciones perinatales y las anomalías congénitas presentaron las tasas más altas. Para la reducción de la alta cifra de este indicador, Kaempffer y Medina (2000) explican que resulta necesario extremar los cuidados prenatales, controlar la nutrición de la embarazada, incrementar la educación de la salud y modificar los factores de riesgo prenatales. 
Por otro lado, Márquez (2006) afirma que "cada año nacen, aproximadamente, 20 millones de bebés de bajo peso, sea por prematuridad o por desarrollo prenatal de alto riesgo; ello contribuye determinantemente a elevar la tasa de mortalidad y morbilidad neonatal"(s.p). Aunado a lo anterior, muchos de los sucesos perinatales suelen causar partos prematuros, los cuales inciden de forma directa en la mortalidad infantil. Tal y como lo describe la Organización Mundial de la Salud (Bryce, 2005), "la mortalidad infantil en los niños menores de 5 años durante el periodo del 2000 al 2003 fue de 10,6 millones de muertes por año; de estas e, el 10\% eran niños prematuros"(s.p). Además, Beck et al. (2010) aseguraron que "los nacimientos prematuros constituyen un 10\% de todos los partos anuales en el mundo". No obstante, el desarrollo paralelo en tecnología y los avances en cuidados obstétricos y neonatales han permitido un incremento importante de la supervivencia de niños prematuros, los cuales son extremadamente inmaduros.

Con respecto a la distribución de la mortalidad según el sexo, se evidencia más casos de muertes de personas de sexo masculino en la mortalidad infantil y, fundamentalmente, en el componente mortalidad neonatal temprana.

Campa (1995) menciona que diversos estudios han reportado que la mortalidad en el sexo masculino ha sido hasta 1,5 veces superior que la del sexo femenino. Explica también que, entre otras razones, el aumento de los niveles de testosterona durante el último trimestre de embarazo causa que los recién nacidos masculinos sean más vulnerables a la asfixia y a la membrana hialina. En nuestro país, no deja de ser diferente, pues se mantiene una TMI más alta en los niños que en las niñas.

Todo lo anterior demuestra cómo los sucesos perinatales significan un reto para la nación y, en específico, para el personal de salud que interviene en el estado de la mujer grávida y del recién nacido.

En el año 2000, el Centro Latinoamericano de Perinatología y Desarrollo Humano realizó un estudio de mortalidad perinatal en las Américas, en el cual Costa Rica mostró una tasa de mortalidad perinatal de 20 por cada 1000 nacidos vivos; esta fue solo superada por Cuba y Chile con 15 por cada 1000 nacidos vivos.

Por consiguiente, como lo señalan Osorio y Romero (2005)

...la calidad de la atención durante e inmediatamente después del período del trabajo de parto y del parto, es la más simple e importante intervención para prevenir la morbimortalidad materna y del recién nacido, y como lo alude Osorio y Romero (2005) la mayoría de los daños obstétricos y los 
riesgos para la salud de la madre y del niño pueden ser prevenidos, detectados y tratados con éxito mediante la aplicación de procedimientos normados para la atención, entre los que destacan el uso del enfoque de riesgo y la realización de actividades eminentemente preventivas y la eliminación o racionalización de algunas prácticas que llevadas a cabo en forma rutinaria aumentan los riesgos(p.722).

De acuerdo a Oliveros (2004)

...es conveniente efectuar estudios a nivel nacional, que nos permitan conocer en detalle la etiología y precisar las estrategias de salud que se adecuen a nuestras necesidades. Finalmente, cabe señalar que la implementación de políticas y protocolos de bajo costo como reforzar la atención prenatal y el entrenamiento del personal de salud en reanimación neonatal, el método canguro, lactancia materna exclusiva son estrategias que están siendo usadas universalmente con excelentes resultados (s.p.)

Lo anterior insta a continuar mejorando la práctica de la Enfermería Ginecoobstétrica y Perinatal y convertirla en una estrategia para la intervención de la población en riesgo; esto último con la finalidad de disminuir la mortalidad infantil y prevenir sucesos perinatales desencadenantes de esta. Por lo tanto, la calidad y eficacia de los cuidados perinatales, que incluyen la vigilancia prenatal y la asistencia intraparto, son acciones para que todo embarazo y parto culmine con una madre y un hijo sanos. Además, al ser la mortalidad perinatal en Costa Rica la principal causa de muerte infantil, resulta imperativo plantearla como uno de los problemas prioritarios en salud pública y sobre el cual resulta necesario intervenir para lograr una reducción significativa de este indicador. 


\section{Revista Electrónica Enfermería Actual en costa Rica}

\section{CONCLUSIONES}

Mundialmente, las causas de mortalidad infantil son diversas: en ocasiones estas van de la mano con problemas en el ambiente o en la infraestructura socioeconómica y sanitaria de la sociedad. También se las asocia con aspectos biológicos individuales, de la salud en general y de la salud sexual y reproductiva, en particular. Por ello, lo relacionado con la mortalidad infantil no escapa del desarrollo social, convirtiéndose en un indicador de desarrollo de vital importancia para cada país.

En Costa Rica, durante el periodo del 2006-2010, la mortalidad infantil se expresa por causas perinatales, en su mayoría. Como principales factores de riesgo, se visualizan los trastornos respiratorios, los cardiovasculares y aquellos relacionados con la duración de la gestación y el crecimiento fetal; estos incluyen el Síndrome de dificultad respiratoria y la prematuridad.

Lo anterior señala que la atención en salud debe enfocarse, especialmente, en esta área; esto implica un reto para el profesional en Ginecoosbtetricia que interviene en la salud de la mujer, del feto o del recién nacido.

\section{REFERENCIAS}

American Psychological Association. (2006). Publication manual of the American Psychological Association. (6th ed.) Washington, DC: American Psychological Asociation

Ávila, M. (2007). Mortalidad Infantil, indicador de calidad en salud. Acta Médica Costarricense.

Beck, S, Wojdyla, D., Say, L., Betran, A., Merialdi, M., Harris, J., Craig, R., Ramkumar, M. y Van Look, P.(2010) Incidencia mundial de parto prematuro: revisión sistemática de la morbilidad y mortalidad maternas. Recuperado de http://www.who.int/bulletin/volumes/88/1/08-062554-ab/es/index.html.

Bryce, J., Boschi-Pinto, C. \& Shibuya K. (2005) RE; WHO Child Health Epidemiology Reference Group. WHO estimates of the causes of death in children? Lancet. , 365(9465), 1147-52

Campa, C., Martínez, C., Russell, G y Acosta, V. (1995). Algunos factores de riesgo de la mortalidad infantil, en un área de salud. Revista Cubana de Medicina General Integral. Recuperado de http://bvs.sld.cu/revistas/mgi/voll1_2 95/mgi19295.htm

Centro Latinoamericano de Perinatología y Desarrollo Humano. (2000). Salud Perinatal. Recuperado dehttp://www.clap.ops-oms.org/web_2005/BOLETINES\%20Y\%20NOVEDADES/BOLETIN18.pdf

Instituto Costarricense de Estadística y Censo. (2011). Mortalidad Infantil y Evolución Reciente. Recuperado de http://unpan1.un.org/intradoc/groups/public/documents/icap/unpan047574.pdf 
Kaempffer, A. y Medina, E. (1998). Análisis de la mortalidad infantil y factores condicionantes: Chile 1998. Rev. chil. pediatr. [Online]. 2000, 71(5) [citado 2012-09-03], pp.405-412. Recuperado de $<$ http://www.scielo.cl/scielo.php?script=sci_arttext\&pid=S0370-41062000000500005\&lng=es\&nrm=iso $>$ ISSN 0370-4106. doi: 10.4067/S0370-41062000000500005.

Márquez, B. (2006). Nivel de conocimientos y actitudes de las enfermeras del servicio de intermedios neonatal del instituto especializado materno perinatal acerca del método canguro 2004 - 2005. Recuperado de http://www.cybertesis.edu.pe/sisbib/2006/marquez bf/html/sdx/marquez bf.html

Mejía, H. (2000). Factores de Riesgo para la muerte neonatal. Revista Sociedad Boliviana de Pediatría 39(3), 104-117. Recuperado de www.ops.org.bo/textocompleto/rnsbp00390303.pdf

Ministerio de Planificación Nacional y Política Económica (2011) Salud: Mortalidad Infantil Mideplan. Recuperado de http://mideplan.go.cr/sides/social/05-13.htm

Oliveros, D. (2004). Mortalidad perinatal en el Perú. Revista Diagnóstico. Recuperado de http://www.fihudiagnostico.org.pe/revista/numeros/2004/ene-feb04/editorial.html

Organización Mundial de la Salud. (2005). Los recién nacidos cobran más importancia. Informe de la Salud en el Mundo 2005. Recuperado de http://www.who.int/whr/2005/es/

Osorio, A. y Romero, V. (, 2008). Factores de riesgo asociados a mortalidad neonatal precoz. Hospital General "Dr. Gustavo A. Rovirosa Pérez," Tabasco, 2005. Salud en Tabasco, 14(1-2), 721-726. Recuperado de http://redalyc.uaemex.mx/src/inicio/ArtPdfRed.jsp?iCve=48711537002

Ticona, M. y Huanco, D. (2005). Mortalidad Perinatal Hospitalaria En El Perú: Factores De Riesgo. Rev. chil. obstet. ginecol., 70(5), 313-317. ISSN 0717-7526. Recuperado de http://www.scielo.cl/pdf/rchog/v70n5/art06.pdf doi: 10.4067/S0717-75262005000500006.

Vargas, M. (2001). Análisis de la mortalidad infantil en Costa Rica. Tesis de Maestría en Epidemiología. Universidad Nacional de Costa Rica. 